\title{
DER EDELE MORINGER.
}

Ein ritter, welcher eine fahrt in weite ferne antreten will, nimmt beim abschiede seiner frau das versprechen ab, eine bestimmte zeit seiner zu warten. Ist dio frist verstrichen, so mag sie einen andern zum manne nehmen. Länger als er gewollt bleibt der ritter aus. Schon steht der tag vor der ture, an welchem die frau ihrer verpflichtungen gegen ihn ledig wird, als er erfährt, dass sie sich wirklich mit einem andern vermählen wolle. Da wird er auf ubernaturliche weise plötzlich in seine heimat versetzt. Unerkannt tritt er in seine burg ein, wo er die ungeduldige gemahlin mit ihrem brăutigam beim hochzeitschmause trifft. Er spielt ihr seinen trauring in die hănde; die erkennung erfolgt, und reuig kehrt sie in die arme des heimgekommenen gatten zurück.

Das sind die grundztge einer sage, welche sich in Deutschland vom mittelalter bis auf die gegenwart in lebendiger tuberlieferung fortgepflanzt hat und bald dieser, bald jener geschichtlichen oder ungeschichtlichen personnlichkeit angeheftet wurde. Deutsche dichtungen thermittelten den stoff den Danen und Schweden sowol wie den Böhmen, die ihn ihrerseits dann weiter nach Russland und nach Ungarn trugen. Aber auch in den romanischen ländern taucht dieselbe sage auf: in Frankreich, in Spanien und in Italien, wo Boccaccio sie der novelle vom Messer Torello (Decamerone IX, 10) zu grunde legte.1)

Dass man den ursprung auch dieser tradition auf irgend einen altgermanischen mythus zurtckzufubren suchte, kann bei

1) Ueber die einzelnen versionen der sage $\nabla g l$. besonders $\nabla$. Tettau, Jahrbücher der kgl. akademie gemeinnitziger wissenschaften zu Erfurt N. F., Heft VI (1870), s. 243-91, wo auch die weitere literatur anfgeführt wird.

Beiträge zur gesohichte der deutschen sprache. XII. 
dem hineinspielen des ubernaturlichen in dieselbe nicht eben befremden. Wolf verglich ${ }^{1}$ ) mit der erzählung von der wunderbaren heimkehr des ritters eine stelle bei Saxo Grammaticus (ed. Muller I, 40), an welcher berichtet wird, dass ein einăugiger greis, d. h. Odin, den fliehenden Hading unter seinen mantel nimmt und ihn so auf seinem pferde mit sich ubers meer fuhrt. Er schloss daraus, der gute oder böse geist, welcher in den verschiedenen versionen unserer sage den helden heimbringt, sei ursprünglich Wuotan gewesen. Wilhelm Müller ${ }^{2}$ ), welcher gegen Wolfs auch von Jac. Grimm vertretene auffassung polemisiert, kommt zu dem resultate, dass vielmehr der heimkehrende selbst eigentlich Wuotan sei, und dass der fahrt in den osten ein mythus von Wuotans fahrt in die unterwelt zu grunde liege. Aber Muller beobachtet dabei im grunde dasselbe verfahren, welches er bei Wolf nissbilligt. Wenn er Wolf vorwirft, dass dieser die ubernaturliche rtickkehr allein behandele, den sonstigen inhalt der sage aber nicht berlicksichtige, so lässt er seinerseits wider jenes von Wolf bevorzugte motiv ausser acht, welches doch zweifellos einen wichtigen und besonders charakterischen bestandteil der ganzen tradition bildet. Fuhrt uns Wolfs untersuchung auf das ziemlich weit abliegende gebiet von erzäblungen wie Fausts oder Ofterdingens luftreise, so lenkt Muller auf eine nicht minder entlegene fabelei des Saxo (I, 42) von dem durch einen zauberer verdrăngten und schliesslich in seine rechte wider eingesetzten Odin ab, welche ubrigens nicht einmal germanischen sondern orientalischen ursprungs ist, wie ich bei anderer gelegenheit nachzuweisen hoffe. Dass auch der von Wolf behandelte teil der sage nicht auf einen deutschen mythus zurtuckgefubrt $\mathrm{zu}$ werden braucht, sondern mindestens ebensowol aus biblisch-apocryphen erzählungen abgeleitet werden kann, hat v. Tettau a. a. o. dargetan, indem er besonders die geschichte von dem durch den engel nach Babylon entfuhrten Habacuc und eine ăhnliche erzăhlung von den aposteln in Marien himmelfahrt vergleicht. Aber wie man auch uber den ursprung dieser einzelnen sagenelemente denken mag, sicher

1) Beiträge z. deutschen mythologie 1, 4 ff.

2) Niedersächsische sagen von Schambach und Müller s. $389 \mathrm{ff}$. 
ist fur den oben aufgestellten, weit verbreiteten typus eben die verbindung derselben charakteristisch, und sie darf vor allem nicht ausser acht gelassen werden, wenn man das zunächst und unzweifelhaft zusammengehörige festhalten, entferntere und unsichere beziehungen aber ausschliessen will.

Die älteste aufzeichnung der hier demnach allein in betracht $\mathrm{zu}$ ziehenden tradition liegt in den 1219/22 verfassten dialogi miraculum des Cäsarius von Heisterbach vor (rec. Strange vol. II, VIII, 59).

In dem dorfe Holenbach, so ungefăhr berichtet Cäsarius auf grund mündlicher uberlieferung, wohnte ein ritter namens Gerardus, dessen enkel noch am leben sind. Er liebte den heiligen apostel Thomas so inbrunstig und ehrte ihn so vor allen andern heiligen, dass er niemand ein almosen abschlug, der ihn in st. Thomas namen ansprach. So kam denn auch einmal ein pilger und bat den Gerardus um st. Thomas willen ihm nachtherberge zu gewähren. Der ritter erfullte nicht nur die bitte, er gab dem fremdling auch noch fir die nacht einen schönen gefutterten mantel. Am andern morgen aber war der pilger mitsamt dem mantel verschwunden - es war nămlich niemand anders als der teufel gewesen, welcher dem frommen Thomasverehrer einen streich spielen wollte. Aber weder durch diese erfahrung noch durch die vorwturfe, welche seine frau ihm uber seine leichtgläubigkeit machte, liess sich Gerardus im glauben an seinen lieblingsheiligen beirren. Er fasste sogar bald darauf den entschluss, zur kirche des heiligen Thomas zu wallfahren. Beim abschiede von seiner gattin zerbrach er einen goldenen ring, gab ibr die eine und behielt sich die andere hălfte: das möge dereinst ein erkennungszeichen fur sie sein. Funf jahro solle sie soiner warten; sei er dann noch nicht zuruckgekehrt, so könne sie einen auderen heiraten. Nach einer langen und beschwerlichen reise kam Gerardus in st. Thomas land an und ward daselbst von den burgern aufgenommen wie ein guter bekannter. Dann trat er in die kirche des heiligen und verrichtete sein gebet. $\mathrm{Da}$ fiel ihm plötzlich ein, dass gerade an diesem tage die 5 jahre um seien, welche er sich von seiner frau ausbedungen hatte. 'Ach', rief er seufzend aus, 'heute nimmt mein weib einen andern zum manne'. - Als er sich umschaut, sieht or jenen betrtige- 
rischen pilgrim in dem gestohlenen mantel dastehen, der sich ihm nun als teufel zu erkennen gibt und ihm mitteilt, dass er den auftrag babe, ibn sofort in die heimat zurlickzuschaffen. Alsbald hob er den Gerardus empor, und noch an demselben abend befand sich dieser vor seinem hause, in welches er wie ein fremdling eintrat. Da sass die frau mit dem brăutigam beim schmause. Gerardus trat heran und liess die hălfte seines ringes in ihren becher fallen. Sogleich erkannte sie ihn, warf sich ihm in die arme und gab dem bräutigam den abschied. Doch behielt ihn Gerardus ehrenhalber noch die nacht dort.

Das charakteristische merkmal dieser version ist die verknupfung des apostel Thomas mit der sage, die dadurch die besondere form der christlichen legende erbält. Die ganze erzählung wird uberhaupt nur als beispiel für den nutzen des Thomascultus vorgebracht, ist also in den dienst einer ganz bestimmten kirchlichen tendenz gestellt. Sie findet sich in dieser gestalt nicht bei Cäsarius allein.

Das stluck einer deutschen 'Legende vom zwölfboten Thomas', welches Zingerle, Zeitschrift f. d. mythologie 4, 39 ohne nähere angaben mitteilt, ist eine ubersetzung desselben textes mit unbedeutenden kurzungen. Und zwar gehört diese ubersetzung dem grossen deutschen prosa-Passional an, in welchem sie den schluss des dem apostel Thomas gewidmeten kapitels bildet. Die legenda aurea, sonst die quelle desselben, enthălt diesen teil der Thomaslegende nicht. Sie enthălt auch nicht die im deutschen Passional unmittelbar vorangehende erzahlung ther die translation des heiligen von Indien nach Syrien und das dort errichtete wunderreiche Thomasmunster, dasselbe, zu welchem nach dem zusammenhange der hier gebotenen uberlieferung jener fromme ritter wallfahrt. Da auch Cãsarius von der translation und dem Thomasmunster nichts berichtet, so mag es zweifelhaft sein, ob das deutsche Passional die darauf beztiglichen mitteilungen zusammen mit der bei Cåsarius vorliegenden geschichte des Gerardus irgend einer dritten quelle entnahm, oder ob es den mit Cäsarius ubereinstimmenden abschnitt doch diesem direct entlehnte. Es gentigt festgestellt zu haben, dass die legende vom Thomasver- 
ehrer Gerardus genau so, wie sie Cåsarius erzăhlte, auch in deutscher ubertragung verbreitet ward.

Unter allen den ubrigen deutschen und ausserdeutschen versionen des im eingange aufgestellten sagentypus aber gibt. es nur eine einzige, in welcher der apostel Thomas eine rolle spielt: es ist das volkslied, oder besser spielmannslied, $\nabla 0 \mathrm{~m}$ edelen Moringer. ${ }^{1}$ )

Der edele Moringer will eine wallfahrt in sant Thomas land tun; bei der abreise verpflichtet er seine frau sieben jahre auf ihn zu warten, befiehlt sie der besonderen obhut seines dienstmannes, des jungen herrn von Neifen, und zieht im vertrauen auf des heiligen Thomas hulfe von dannen (nachdem er beim abschiede noch einen ring ron ihr empfangen hat: hs. C und Zimm. Chron.). Er erreicht sein ziel; aber schon sind die sieben jabre um, als er noch in st. Thomas land weilt. Da hört er im schlafe eine stimme vom himmel: 'Wach auf Moringer! kommst du nicht heute abend noch heim, so nimmt sich der junge von Neifen dein weib'. Jammer und ingrimm erfasst den edlen Moringer und er klagt dem heiligen Thomas sein bitteres leid. Dann entschlaft er wider, und als er orwacht, befindet er sich plötzlich in seiner heimat. Als pilger klopft er an die pforte seiner eigenen burg und erbittet sich

1) Uhlands Volkslieder nr. 298. Anmerkungen und lesarten dazn in Uhlands schriften IV, $286 \mathrm{ff}$. Da die bekannten handschriften und alten drucke des Moringerliedes noch nirgend ganz vollstindig zusammengestellt sind, so mogen sie hier kurz aufgefuhrt werden: 1. Handschriften: $A=$ Veesenmeyersche auf der Ulmer stadtbibliothek, v. j. 1459. (Die auf schreibfehler beruhende, schon von Uhland a. a. berichtigte datierung 1359 wird noch bei Wackernagel L. G. I' s. 180 anm 13, Bartsch, Herzog Ernst CX, Bartsch-Koberstein I' 350 irrtumlich zur zeitbestimmung des liedes verwertet). $-B=$ Thomanns Chronik in verschiedenen hss., vgl. Baumann, Quellen z. gesch. d. bauernkrieges s. $235 \mathrm{ff}$., nach einer dieser hss. abgedruckt in Gräters Bragar III, 403. - $\mathrm{C}=$ Wernigerode, gräfl. Stolbergische bibliothek, sign. Zb $4 \mathrm{~m}$, 8. 249b/55 b (stark abweichender und verderbter text). - - Il. Drucke: a) Bamberg 1493, abgedruckt Bragur VIII, 200. -- b) Erfurt 1497 (in einem sammelbande des archivs der Marienkirche daselbst). - c) Erfurt 1500. - d) Nurnberg (A. Dyon) um 1510 (k. bibliothek, Berlin). - e) Nürnberg (Gutknecht) um 1515. - f) Ohne titel um 1570. - g) 0. 0. (Basel, Schröter) 1605. - Ueber die drucke vgl. besonders v. Tettau a. a. o. Uhland benutzte $B, a, d, f$. Die späteren abdrücke gehen auf Uhland zurïck. 
ein almosen um gottes und sanct Thomas willen und um des edlen Moringers ehre. Um gottes und sanct Thomas willen wird er auf geheiss seiner frau freundlich eingelassen, die wirklich gerade mit dem jungen herrn von Neifen hochzeit feiert. Als braut und bräutigam zu bette gehen wollen, fordert man den pilgrim auf, zuvor noch ein hoveliet $\mathrm{zu}$ singen; denn das habe der Moringer von jedem gaste verlangt, der bei ihm zur nacht bleiben wollte. So hebt er denn an; und sein lied ist uns ein alter bekannter: es sind zwei strophen eines gedichtes Walthers von der Vogelweide (Lachm. 72, 31), welche hier trefflich der situation angepasst wurden. Der sänger klagt, dass seine frouwe ihm einen jungen vorziehe, und bittet diesen in derber weise, ihn an der alten braut zu rächen. Als die frau das hört, wird sie betrubt; zum lohne für den gesang aber lässt sie dem pilger einen becher weines reichen. Der lässt seinen trauring hineinfallen, und als die dame so den becher zurückerhält, da erkennt sie den heimgekehrten gemahl. Sie wirft sich ihm reuig zu fussen; der junge herr von Neifen ist bereit seine treulosigkeit mit dem kopfe zu bezahlen. Doch der Moringer verzeiht ihm und gibt ihm sogar seine tochter zum weibe; dagegen will er selbst die alte braut nun fur sich behalten.

Das ist der hauptsache nach der inhalt des Moringerliedes, welcher also durch das hereinziehen des heil. Thomas der erzăhlung des Cåsarius zweifellos am nächsten verwant ist. Die besondere, in schriftlicher und mundlicher taberlieferung verbreitete version der sage, welche wir einfach die Thomaslegende nennen können, bildet auch die grundlage unseres spielmannsliedes. Dass dieses nebenbei in manchen punkten von Cåsarius abweicht, ist ein umstand, der an jener tatsache nichts ändert. Der teufel, der wie bei Cäsarius so auch in manchen anderen versionen die ruckbeförderung des ritters ubernehmen muss, wird hier aus dem spiele gelassen; das wunder der heimkehr bleibt eben unerklärt. Wichtiger als der fortfall dieses manchem vielleicht anstössigen zuges der sage ist die verbindung derselben mit neuen, weder bei Cäsarius noch in anderen versionen nachweisbaren namen. Vor allem fragt es sich: wer ist dieser edele Moringer? wer ferner der junge herr von Neifen? Wie kam man dazu, jene verbreitete 
sage und zwar die sage in der besonderen form der Thomaslegende gerade auf den Moringer zu ubertragen? und wie erklärt sich die einfugung eines liedes des Walther von der Vogelweide in diesem wunderlichen zusammenhang?

Die beantwortung der ersten beiden fragen und wenigstens eines teiles der dritten ist schon vielfach versucht worden, ohne dass eine befriedigende lōsung dabei erzielt wäre. Wackernagel meinte, hinter dem namen Moringer, Maringer stecke vielleicht ein appellativum, nämlich 'meringer d. h. marinaro, seefahrer' (Lit. gesch. ${ }^{2}$, s. 180 anm. 13), eine vermutung, die von andern mit grösserer bestimmtheit widerholt wurde (Goedeke, D. dicht. i. ma. s. 576; Böhme, Altd. liederb. s. 37). Aber einmal ist ein wort meringer = seefahrer nirgend belegbar, eine solche bildung auch sprachlich mindestens höchst unwahrscheinlich; dem italienischen marinaro entspricht allein das von Wackernagel selbst herbeigezogene marner. Sodann ist der held des gedichtes niemals Meringer genannt worden, in den bekannten hss. und drucken desselben nicht einmal Möringer sondern nur Moringer oder Morgener, welche letztere form auch nur aus der umlautlosen Mornger hervorgegangen sein kann. Wir können also von jener erklärung völlig absehen.

Als eine historische persönlichkeit suchte Stälin, Wirtemberg. gesch. II, 575, den Moringer nachzuweisen. Berthold III., herr von Neifen, der zuerst in einer urkunde v. j. 1241 auftritt, heiratete Gottfrieds grafen von Marstetten erbtochter Jutta und erbielt dadurch wurde und namen eines grafen von Marstetten. 'Die erwerbung der grafschaft Marstetten durch Berthold von Neifen gab den stoff zu folgender sagenhaften erzäblung: Der edle Moringer (diesen namen setzt die dichtung an die stelle des grafen von Marstetten), 7 jahre auf einer wallfahrt nach Indien abwesend', kommt schliesslich unter den oben erzählten umständen zurtuck, wird mit seiner gattin wider vereint und gibt 'dem herrn von Neifen zur entschädigung die hand seiner erbtochter'. - Wie die sage dazu gekommen sein soll, den grafen Gottfried von Marstetten den edlen Moringer zu nennen, ihm eine wallfahrt in st. Thomasland ${ }^{1}$ ),

1) St. Thomasland ist gewöhnlich Indien, wo Thomas nach der 
jene wunderbare heimkehr von derselben und die vereitelung der ehe seiner frau mit dem herrn von Neifen zuzuschreiben, alles das bleibt unaufgeklärt; und das ist im grunde der ganze inhalt des Moringerliedes. Der einzige vergleichungspunkt, der sich nach Stălins inhaltsangabe ergeben wüde, wåre die verbindung der 'erbtochter' mit dem herrn von Neifen. Aber von einer erbtochter weiss das Moringerlied selbst gar nichts. 'Habent euch die tochter mein, und laszent mir die alte braut', sagt der Moringer am schluss zu dem jungen herrn von Neifen; und auf diesen einen vers, welcher nur eine andere wendung des schon bei Căsarius vorliegenden versöhnlichen ausganges ganz nebenbei bringt, ist Stălins ganze combination gebaut.1) Dass man mit demselben rechte wie die verehelichung des Berthold von Neifen mit jener gräfin von Marstetten auch die verheiratung irgend eines beliebigen andern herrn von Neifen als den ausgangs- oder anknlipfungspunkt der tradition hinstellen könnte, ist klar, und es wäre geradezu unbegreiflich, wie Stălin zu einer derartigen aufstellung kommen konnte, wenn nicht doch noch ein anderer, wenigstens scheinbarer anhalt für dieselbe existierte, welchen Stälin gewiss gekannt, aber nicht erwăhnt bat. Es ist das eine nachricht, die Nikolaus Thomann in seiner im jahre 1536 vollendeten Weissenborner chronik bringt. Eine ungenaue mitteilung gab dartber Schmid in Gråters Bragur III, $402 \mathrm{f}$; sie kann jetzt, wo eine ausgabe jener chronik in Baumanns Quellen zur geschichte des bauernkrieges s. 1 ff. vorliegt, die erforderliche ergänzung erfahren. Thomann erzahlt, er habe in einem sehr alten kalender die notiz gefunden, dass ein Berchtoldus von Neuffen am XX. februar 1349 und dass eine gräfin Elisabeth v. Neuffen am tage Matthiae (das war der 24. februar) gestorben sei. Das todesjahr der letzteren war nicht angegeben. Weytter hab ich gefunden in dusem kalender, das fraw Elisabet von Neuffen,

legende als apostel wirkte und den märtyrertod erlitt. In der hier 7. a grunde liegenden tradition aber kann nach s. 434 auch Syrien gemeint sein. Der verfasser des liedes hat von seinem Thomasland wol nichts weiter gewusst, als dass es im orient liegt.

1) Bartsch, Herz. Ernst 8. CXV und Kobersteins Grundr. I $^{6}$ s. 350 sieht dieselbe gleichwol als zweifellos festgestellt an; ebenso Holland in Uhlands schriften IV, 297 und Barack, Zimmerische chronik $\mathrm{I}^{2}$ s. 300 anm. 
gräfin zu Marstetten, die man genent hat die Moringerin, die jarzal ires absterbes stunt ach nit dabey, aber am XI. tag des monetz May ist sy gestorben. Thomann vermutet, dass diese drei in Weissenhorn (bair. Schwaben) begraben seien, weil jenes calendarium dorthin gehört und făhrt dann fort: Esz ist zu vermercken, das durch gar alte sagen und aynes alten lieds angezaygt wurt, wie das ain edler her zu Buch gesessen sey, der hab gehayssen Moringer. der sey bulgerisz weys in sant Thomas lant gezogen und sein gemachel, lant und lewt aynem jungen herren und grafen von Newffen bevolhen und sey suben jar ausbeliben. wie und in welcher gestalt er widerumb zu lant komen sey, wurt in dem nachfolgenden liet anzaygt. ob esz also geschen und ergangen sey, dabey bin ich nit gwesen, wulsz ach nit weytter erkunnen (folgt das von Baumann nicht mitgeteilte, von Schmidt a. a. 0. abgedruckte lied). Item nach anzaygen duses lyeds oder geduchs musz der edel Moringer lang vor dienen von Neuffen gwesen sein und die von Neuffen durch den Moringer daher komen sein. - Es ist nit gruntlich davon ze schreiben, aber von alter her fur und fur ist von duser histori gesungen und gesayt worden und insunder zu Buch. - Anzaygen - Esz ist da vornen anzaygt von fraw Elisabet von Neuffen, grefin zu Marstetten, dieselben hat man genent die Moringerin, sy kann aber nit des Moringers dochter gewesen sein, sy möchte aber woll oder fulleicht zu ayner gedechtnusz irer voreltern also genempt worden sein.

Hier haben wir also in der tat eine vereinigung der namen Marstetten, Moringer und Neuffen in einer person und damit zugleich scheinbar einen anhalt fur Stälins combination. Auch von Tettau folgert daraus nicht allein die zusammengehörigkeit der namen Neifen-Marstetten und Moringer, sondern auch die identität des grafen Gottfried v. Marstetten mit dem edelen Moringer und die der erbtochter jenes Gottfried mit der dem jungen Neifen versprochenen tochter des Moringers. Diese folgerung ist aber nur möglich, wenn man voraussetzt, dass die Moringerin der Thomannschen chronik eben jene tochter Gottfrieds von Marstetten gewesen sei, welche Berthold von Neifen heiratete, eine annahme, welche wol gestattet war, so lange man von Thomanns Moringerin nur durch die oben erwähnte mitteilung Schmids wusste, der den vornamen dieser 
frau von Neifen-Marstetten, genannt Moringerin, nicht mitteilte. Jetzt aber sehen wir, dass diese letztere Elisabeth, die erbtochter Gottfrieds von Marstetten aber Jutta hiess, und damit wird jene folgerung hinfällig. Da die grafschaft Marstetten durch Juttas ehe mit Berthold an die herrn von Neifen ubergieng, so muss die Moringerin Elisabeth von Neifen und Marstetten nach der Jutta gelebt haben. Sie wird also die frau eines nachkommen des Berthold und der. Jutta und als solche frau v. Neifen und grăfin von Marstetten gewesen sein. Den beinamen Moringerin mochte sie von einem der schwäbischen oder bairischen orte Moringen oder Möringen haben, aus dem sie vielleicht herstammte; etwas sicheres wird sich darubber schwerlich ermitteln lassen. Jedenfalls existiert nach alledem auch nicht mehr die geringste berechtigung, das Moringerlied auf den ubergang der grafschaft Marstetten an die herrn von Neifen zu beziehen und den Moringer für den grafen Gottfried von Marstetten oder einen andern dieses geschlechtes zu halten. Die einzige bedeutung, welche die Thomannsche notiz fur die sage vielleicht haben könnte, ist die, dass jene Elisabeth von Neifen und Marstetten, genannt Moringerin, möglicherweise den anlass gegeben hätte, den namen von Neifen mit einer schon feststehenden sage vom edlen Moringer in verbindung zu bringen. Ueber den Moringer selbst aber gewinnen wir daraus gar keinen aufschluss.

Andere nachrichten gibt uber den belden unseres gedichtes die Zimmerische Chronik (Barack I, 286 ff. I2, 300 ff.): Aber den eltesten landfarer, den wir in unsern hochen deutschen landen gehapt, darvon wir noch wissent, das ist der edel Moringer gewesen. Denselbigen wellen ellich, er seie ein Meichsner oder ain Sax gewesen. Gleichwol auch ainer vor jaren mag gelept (haben) so der Moringer hat gehaiszen, soll zu Leipzig gesessen und in groszem thon gewesen sein, wie man furgibt, aber dieser unser Möringer, ist ain Schwab gewesen und ain mechtiger landsherr; er hat sein haimwesen zu Munderkingen an der Tonaw auch uf und umb den Bussen gehabt; gleichwol man sein geschlecht aigentlichen nit waist, aber vermutlichen so ist er ain graf des herkommens von Hapspurg oder hat doch vast ain gleichfermigs wappen gehabt. ... Mun sagt, er hab den namen vom stetlin Meringen an der Tonaw bekommen, alldo sei er geporen 
worden, welches von alter nit Möringen gehaiszen, sonder Moringen. ${ }^{1)}$ Das bezeucht des stetlins wappen und sigel, das sie von unverdechtlichen jaren hergebracht mit dem morenkopf. Nun diser Moringer, er habe gleich gehaiszen oder sei ains geschlechts gewest wie er welle, ... hat ain weib gehabt aines furnemen geschlechts, und von deren schöne und frombkait vil wurt in liedern gesungen. In sollichem glucklichen zustande ... ist im ... zu sinn kommen, das er den hailigen apostel sant Thomassen, den er all sein tag vorder in ehren gehapt, in India solle haimsuchen - und nun erfolgt eine ausfuhrliche inhaltsangabe des Moringerliedes ${ }^{2}$ ) mit mancherlei ausschmlickungen und starker änderung einiger der vom Moringer gesungenen verse. Zum schluss folgt noch die angabe, wann der Moringer gestorben sei, wisse man nicht, aber seine rennfahne mit verblichenem wappen sei noch vor wenigen jahren vorbanden gewesen und zwar im besitz einer frau Veronika Spettin zu Freiburg im Breisgau.

Mit Thomanns Chronik stimmt dieser bericht nur insofern uberein, als der Moringer auch hier für einen schwăbischen ritter ausgegeben wird. Die bestimmteren örtlichkeiten aber, mit welchen diese personnlichkeit verknupft wird, sind in den beiden chroniken verschieden. An die grafen von Marstetten, deren stammburg an der mittleren Iller lag, haben die verfasser der Zimmrischen chronik jedenfalls nicht gedacht, da sie Munderkingen a. d. Donau und den sudlich davon gelegelegenen Bussen als des Moringers heimat bezeichnen. Noch weiter abseits aber fuhren die beziehungen auf Möringen a. d. Donau, Freiburg i. Breisgau und die Habsburger. Und schliesslich weiss die eine chronik so wenig wie die andere den Moringer selbst historisch nachzuweisen. Sie suchen denselben in Schwaben, weil der herr von Neifen dorthin weist

1) Oesterley, Hist. geogr. wörterbuch s. 452, führt wenigstens für das Möhringen bei Stuttgart und für das bairische Möhring die ältere form Moringen auf. Nibelungen 1531, 1 überliefern die hss. A a b Moringen, die übrigen Mœringen.

2) Als der Moringer in seine burg eintritt, heisst es: er 'war unnutz vol', was Barack in 'unmäss vro' ändern will; es muss vielmehr 'unmuetz vol' heissen; vgl. Moringerlied 27 do der edel Moringer in sein eigen burg eingieng im was leid und also schwär. 
und weil das Moringerlied dort im umlauf ist; die Zimmerische chronik tberdies wegen des schwăbischen Möringen a. d. Donau, die Thomannsche wegen einer gräfin von Neuffen-Marstetten, welche Moringerin genannt ward.

Dabei gibt aber die Zimmerische chronik selbst einen fingerzeig, welcher auf eine ganz andere fährte weist. Es ist die angabe, dass von des Moringers frau, von ihrer schönheit und trefflichkeit, viel in liedern gesungen werde. Die richtige deutung dieser notiz, für welche das Moringerlied nicht den mindesten anhalt bot, hat schon Ludwig Uhland Germ. IV, 95 gegeben, ohne jedoch weitere folgerungen daraus zu ziehen. Der chronist kannte die lieder, welche der minnesinger Heinrich von Morungen zum lobe seiner frouwe verfasst hatte. Wir wissen bestimmt, dass ihm eine handschrift vorlag, welche diese lieder enthielt. Es war eine jetzt verlorene sammlung von minnesingern, deren namen er $\mathrm{II}^{2}, 194$ verzeichnet, darunter auch her Rainhart von Brennenberg, her Hainrich von Morungen, baid ritter. Angesichts dieser lieder, deren erstes nach der uns vorliegenden uberlieferung gleich die schönheit und trefflichkeit der frouwe Morungens begeistert preist, mag er die worte 'es wird viel von ihr gesungen' geschrieben haben; dann aber bat er auch den minnesinger Heinrich von Morungen und den Moringer fur eine person gehalten. Hatte er darin recht, so war es ein irrtum, diese persönlichkeit nach Schwaben zu versetzen. Es steht bekanntlich fest, dass Heinrich von Morungen ein Mitteldeutscher war, und dass die burg Morungen bei Sangerhausen seinem geschlechte den namen gab.1) Das wappen der Sangerhausener Morunger stimmt denn auch noch in seiner aus dem ende des 16. jahrhunderts nachgewiesenen gestalt im wesentlichen uberein mit demjenigen, welches die Pariser liederhandschrift dem minnesinger Heinrich von Morungen beilegt.2) Danach wurde - immer vorausgesetzt, dass die identificierung des Moringers und Heinrichs von Morungen richtig ist - jene andere, von der Zimmerischen chronik ver-

1) Vgl. v. Mülverstedt, Zeitschrift des Harzvereins 13, $440 \mathrm{ff}$. und die $M^{3}{ }^{3}$ 8. 281 angeführte literatur.

2) Vgl. Zurborg, Zeitschr. f. d. altert. 18, 319. Die mitteilung älterer siegelwappen der Sangerhausener Morunger, welche v. Mülverstedt a. a. o. in aussicht stellt, ist meines wissens bisher noch nicht erfolgt. 
worfene nachricht der wahrheit viel năher kommen, nach welcher der Moringer ein Meissner oder ein Sachse gewesen sei. Aber noch mebr: diese nachricht erhălt mitsamt der weiteren angabe, der Moringer sei in Leipzig gesessen und in grossem ton (in grossem ansehen) gewesen, eine ganz merkwürdige bestätigung durch die einzige urkunde in welcher ein Heinrich von Morungen auftritt, der mit dem minnesinger identificiert werden kann. Dieser urkundlich nachgewiesene Heinrich von Morungen steht im dienste des markgrafen Dietrich von Meissen, scheint zur zeit in Leipzig zu leben und befindet sich augenscheinlich in ansehnlichen verhăltnissen, da er einem woltătigen zwecke jährlich eine betrăchtliche summe opfert.

Auf denselben Leipziger Heinrich von Morungen weist nun ferner die wichtige auch von Gottschan Beitr. 7, 338 behandelte mitteilung im wappenbuche des Konrad Grinenberg v. j. 1483. Dort ist ein wappen abgebildet, welches sowol zu dem der Pariser liederhandschrift als zu dem der Sangerhausener Morunger stimmt, ja die formen der beiden gewissermassen vermittelt; und die uberschrift desselben lautet: der Edel Moringer der zuo Lips begraben litt.1) Hier einigen sich also alle beziehungen aufs schönste. Wir erhalten eine neve bestătigung dafur, dass der minnesinger der Pariser liederhandschrift zum Sangerhausener geschlechte gehört und dass or mit dem Leipziger Morunger der urkunde identificiert werden kann. Weiter aber erfahren wir auch, dass Konrad Grtunenberg ebea diese personnlichkeit fur den edlen Moringer, d. b. fur den helden des liedes halt; und damit werden wir wider auf dieselbe tradition zurtlckgefuhrt, welche wir aus den einzelnen angaben der Zimmerischen cbronik ersobliessen konnten: der edele Moringer ist der minnesinger Heinrich von Morungen, welcher einst zu Leipzig in grossem ansehen lebte.

Freilich bleibt bei Granenberg so gut wie bei dem chronisten daneben noch ein platz für einen schwäbischen Mo-

1) So nach v. Mulverstedt a. a. 0. 451; vgl. die Beilsgen daselbst. Die ausgabe des Grünenbergischen wappenbuches durch den grafen von Stillfried-Alcantara (vgl. Gottschan s. 338) enthält nach v. Mtilverstedt Moringers wappen nicht. 
ringer. Denn vor dem eben behandelten wappen bringt Grünenberg noch dasjenige des Hainrich von Moringen: es ist ein mohrenkopf im gelben felde, d. b. ein wappen, welches nach der oben mitgeteilten angabe der Zimmerischen chronik der schwäbischen stadt Möringen oder Moringen a. Donau zukommt, und welches zugleich dem Heinrich von Mornngen in der Weingartner liederhandschrift beigelegt wird. Dass letztere mit diesem augenscheinlich auf den namen Morungen zugeschnittenen wappen gegentuber dem historisch beglaubigten der Pariser handschrift nicht das richtige bietet, ist klar. Grunenberg aber fand beide wappen vor und bezog das eine richtig auf den Leipziger Morungen, während ihn das andere, welches er wol aus der Weingartner handschrift selbst kennen lernte ${ }^{1}$ ), an das gleiche wappen der stadt Moringen erinnerte, weshalb er denn in der uberschrift statt des in jener handschrift tiberlieferten Morungen die form Moringen einsetzte. So gerăt denn hier Grunenberg auf denselben irrweg, auf den sich die Zimmerische chronik verlor, nur dass es sich bei ihm besonders deutlich verfolgen lăsst, wie sich aus der person des historischen Heinrich von Morungen die phantasiegestalt seines schwäbischen doppelgăngers ablöst. Zugleich sehen wir, wie der dichter seinen namen von Morungen gegen den von Moringen oder Moringer eintauscht. Dieselbe erscheinung zeigt sich, wenn er, der wegen seines tageliedes für einen typischen vertreter der minnedibe galt, im Seifried Helbling als solcher mit dem namen Morungaer (Helbling ed. Seemuller I, 760), in einem losbuche des 15. jahrhunderts aber in gleicher eigenschaft als Moringer aufgefuhrt wird. ${ }^{2}$ )

Nach alledem tritt uns aus den verschiedenen alten traditionen uber geschlecht und heimat des edelen Moringers doch nur eine einzige historisch bestimmbare persönlichkeit entgegen: der minnesinger Heinrich von Morungen. Traf die

1) Vgl. Germania 29 s. 488 (zu nr. 1219) und v. Mülverstedt, der a. a. 0 . in der beilage diese wappen einander gegenuiberstellt. Das richtigere wappenbild bei Grinenberg stammte nach v. Mulverstedts vermutung von Heinrichs grabstein, der sich im Thomaskloster zu Leipzig befinden mochte.

2) Die vier puoler Wolfram von Eschenbach, Moringer, Prennberger, Fuoss der puler. Grimm HS 284. 
uberlieferung, welche ihn fur den helden der sage ansah, wirklich das richtige? Wir durften die frage bejahen, wenn sich ein bestimmter anlass erweisen liesse, gerade auf ihn die in redo stebende sage zu tubertragen.

Erinnern wir uns, dass die beziehung des Moringers zum heiligen Thomas fur die besondere version der sage im Moringerliede charakteristisch und für den năheren zusammenhang derselben mit der legende des Căsarius beweisend ist, so gewinnt auch hier das einzige historische zeugnis, welches wir uber Heinrich von Morungen besitzen, bedeutung, jene oben erwăhnte urkunde nämlich, welche sein dienstherr, markgraf Dietrich von Meissen in der zeit nach 1213 und vor 1221 a usfertigen liess. Die beztgliche stelle der mehrfach gedruckten urkunde, auf welche zuerst Bech, Germ. 19, 419 aufmerksam machte, lautet (Cod. diplom. Sax. reg., II. hauptteil bd. IX, p. 7): ... Henricus de Morungen, miles emeritus, spiritu tractus divino, $X$ talenta annuatim, quae propter alta vitae suae merita a nobis ex moneta Lipzensi tenuit in beneficium, nobis resignavit et ut ea ecclesiae beati Thomae in Lipzc ad usus inibi Christo militantium conferre dignaremur, devotissime supplicavit. Der markgraf bewilligt dann dem Thomaskloster fur alle zeiten die stiftung des Heinrich von Morungen unter wärmster anerkennung dieses frommen werkes.

Von Tettau, der noch an der beziehung des Moringers auf den grafen Gottfried von Marstetten festhielt, bemerkte gleichwol ganz richtig im anschluss an die erzählung des Cäsarius vom Gerhard von Holenbach, dass eine gleiche besondere verehrung des heiligen Thomas seitens des einen wie des andern ... die veranlassung gegeben haben möge das ursprunglich von Gerhard erzahlto auf den Moringer zu ubertragen. Nun, in unserer urkunde lernen wir einen Morunger oder Moringer als besonderen verehrer des heiligen Thomas kennen; denn dass seine reichliche schenkung an die kirche dieses heiligen ganz dazu angetan war, ihm diesen ruf zu verschaffen, wird niemand in abrede stellen. Und so scheint sich denn die fur das 15. und 16. jabrhundert nachgewiesene tradition zu bestātigen, nach welcher jener Leipziger Heinrich von Morungen, d. b. der minnesinger, der held unseres gedichtes war; wir haben wirklich einen anlass gefunden, wes- 
halb man gerade auf ihn die legende vom frommen Thomasverehrer thertragen konnte.

Möglich ist es auch, dass Heinrich selbst eine fahrt ins morgenland gemacht hat. Dass er dem Dietrich von Meissen lange jahre hindurch treue dienste geleistet haben muss, können wir aus des markgrafen urkunde entnehmen, die der alta vitae merita des Morungers gedenkt. Dietrich aber unternahm im jahre 1197 eine kreuzfahrt, auf der ihn dennnach Heinrich von Morungen begleitet haben mag. Dass dieses ereignisses in Morungens liedern nicht gedacht wird, spricht nicht gegen jene annahme; denn was uns von Morungens poesie erhalten ist, kann alles vor 1197 gedichtet sein.1) Zur zeit der urkunde, d. h. um 1215, war Heinrich, als miles emeritus, jedenfalls schon ein alter mann (vgl. v. Mulverstedt a. a. o. s. 469 anm. 4). Von dieser kreuzfahrt nun kehrte Dietrich auf die nacbricht rom tode des kaiser Heinrich alsbald wider heim, um die ihm bisher vom kaiser vorenthaltene markgrafschaft Meissen zu besetzen. Und sagenhafte gertchte verbreiteten sich tuber die art seiner ruckkebr. Die chronik des Petersberger klosters bei Halle verlegte das ereignis in das jahr 1196 und erzăhlte, Dietrich babe damals im orient die kunde vom tode seines bruders und gegners, des markgrafen Albrecht erhalten und habe sich alsbald, in einem fasse versteckt, auf das schiff bringen lassen, um so vor den nachstellungen kaiser Heinrichs gesichert heimzukehren (MGSS XXIII, 166; vgl. dazu Opel, Das chronicon montis sereni 8. 23/25). Nahm Heinrich von Morungen an dieser fahrt teil, oder brachte man ihn als Dietrichs dienstmann spăter mit derselben in verbindung, so war damit ein zweiter anlass gegeben, die sage von der orientfahrt und wunderbaren heimkehr des Thomasverehrers auf ihn zu ubertragen. Entscheidende bedeutung lege ich

1) Weissenfels, Der daktyl. rhythmus bei den minnesängern, scheint mir s. 158 aus der besonderen verwendung jenes rhythmus bei Heinrich v. Morungen ohne not eine spätere abfassungszeit fur dessen lieder zu folgern. Er ist sich auch wol selbst noch nicht ganz sicher in dieser annahme gewesen, denn noch auf s. 131 setzt er Morungen unter die vorwaltherischen lyriker. Ueber die verwertung dieser ubrigens sehr verdienstlichen metrischen beobachtungen als grundlage chronologischer bestimmungen vgl. auch Weissenfels s. 3 anm. 3 . 
naturlich dieser immerhin zweifelhaften beziehung nicht bei, nur soviel, um die erwăhnung derselben neben anderen und gewichtigeren grunden nicht fur uberflussig zu balten.

Wenn nun die in rede stehende sage oder legende wirklich seinerzeit auf den minnesinger Heinrich von Morungen ubertragen ward, so wurde es sich doch immer noch fragen, ob auch der jedenfalls viel spătere verfasser der uns vorliegenden poetischen bearbeitung derselben bei seinem edelen Moringer noch an jenen minnesinger dachte ${ }^{1}$ )? Die antwort muss von der erwăgung desjenigen ausgehen, was dem Moringerliede im unterschiede von den anderweitig uberlieferten sagenmotiven allein eigen ist. Dahin gehört die benutzung eines liedes Walthers von der Vogelweide und die einfuhrung des jungen herrn von Neifen. Nun mulsste es doch ein ganz wunderlicher zufall sein, wenn in einem gedichte, welches das lied eines minnesingers benutzt, als einzige personennamen die zweier anderer minnesinger vorkommen, ohne dass der dichter letztere dabei im sinne gehabt hătte. Die vermutung, dass derselbe mit dem Moringer wirklich den Heinrich von Morungen, mit dem jungen von Neifen Gottfried ron Neifen gemeint habe, musste sich von vornherein aufdrăngen; sie ist denn auch schon von Jak. Grimm (Altd. meistergesang s. 184) aufgestellt. Grimm wies mit recht darauf hin, dass der anfang des liedes, welches der Moringer singt ein langes schweigen hab ich gedacht, so wil ich aber singen als $\hat{E}_{\text {. darzu hant }}$ mich die fraven bracht die mugen mir wol gebieten mê nur in den mund eines minnesingers passt. Er verglich damit Heinrichs von Morungen strophe: Waer ir mit mime sange wol, sô sunge ich ir: sus verbôt siz mir, wan ir tuot min snígen baz. nu snige $a b$ ich ze lange: solde ich singen $m \hat{\varepsilon}$, daz tet ich als $\hat{e}$. MF 123, 22 und or bemerkte endlich, dass das versmass des Moringerliedes in einigen liedern Morungens vorgebildet zu sein scheine. Diese grtude konnten denn freilich nicht aus-

1) Scherer verneint diese frage. Er meint 'dass Brennenberg, Morangeu, Neifen, Tannhänser dichter waren, weics das volkolied nicht mehr'. Fur den helden der sage aber hält er (allein unter allen literarhistorikern) trotzdem den Heinrich v. Morungen; freilich ohne einen weiteren grund dafur beizubringen, als den anklang des namens Morungen 'an die mohren und das mohrenland'. LG'. 258. 742.

Beitrage sur geechichte der deutechen sprwahe. XII. 
reichen, um Grimms behauptung zu erweisen, umsoweniger als es sich nachher heraustellte, dass die einzige strophe des Moringerliedes, deren wortlaut ihn an Heinrich von Morungen erinnert batte, einem liede Walthers von der Vogelweide entnommen war. Auch die vergleichung der strophenform Morungensclier lieder traf nicht durchweg zu, blieb indes immerhin bei zweien zu recht bestehen: der ton des liedes frouwe, wilt du mich genern MF. 137, 10 stimmt mit denjenigen strophen des Moringerliedes uberein, welche durchweg stumpf ausgehen; der des liedes hât man mich gesehen in sorgen MF 144, 17 mit denen, welche im 1. und 3. verse klingend schliessen.1)

Erheblich vermehren lassen sich die beziehungen zwischen dem Moringerliede und den liedern des Morungers nicht. An die klage der frouwe uber das scheiden des liebenden MF. 131, 1 und an des letzteren bildlich gemeinte worte ich wil eine reise, wünschel daz ich wol gevar MF. 145, 33 wird man bei den ersten strophen des Moringerliedes, welche den abschied des Moringers von seiner frau behandeln, schwerlich denken durfen. Eher kann man etwa vergleichen frowe, ich kan niht wenken (die reimzeile fehlt) hastu tugende und êren vil, daz wolt ich und immer wil MF 146,15 und Im glauben wil ich euch nit wenken (: gedenken) ... So gsegen euch got, edel frave. in also tugenthaftem mut. aller eren ich euch getrawe Moringerlied 5. 6.

Aber mit keinem liede Heinrichs von Morungen zeigt sich auch nur annăhernd eine solche thereinstimmung wie mit jenem liede Walthers von der Vogelweide (Lachmann 72, 31). Der dichter des Moringerliedes hat demselben nicht nur die beiden strophen entlehnt, welche er seinem belden in den mund legt; das lied hat inm auch sonst vorgeschwebt. Als der Moringer erfăhrt, dass seine frau einen andern nebmen wolle, klagt er dass mich mein fraw wil scheiden von er, die ich han bracht zu wirdigkeit. Aus dem zusammenhange des liedes sind die letzten worte nicht zu erklären. Nirgend findet sich eine andeutung, dass des Moringers frau erst durch ihre verheiratung aus einer niedrigeren in eine hohe stellung

1) Ueber den klingenden ausgang in den Moringerstrophen vgl Bartsch, Herzog Ernst CX f. 
erboben sei. Der vers erhält erst die richtige beleuchtung durch eine parallelstelle in dem erwähnten liede Walthers. Der dichter klagt dort mich enwil ein wip niht angesehen: die brâht ich in die werdekeit, daz ir muot sô hôhe stat. jon weiz si niht, swenn ich mîn singen läze, daz ir lop zergât Walth. 73, 1. Hier ist alles klar. Der sänger hat mit seinen liedern seine frouwe $\mathrm{zu}$ bohem ansehen gebracht, die ihm nun ubel dafur lohnt. Aus diesem zusammenhange stammt offenbar der mit dem Waltherschen ubereinstimmende vers des Moringerliedes, und auch bei dieser entlehnung wird der dichter sich seinen Moringer als minnesinger gedacht haben. Aber nehmen wir an, dass dies der fall war, das heisst nach allem vorangegangenen, dass der dichter mit dem Moringer den Heinrich von Morungen meinte, wio kam er dann dazu, demselben stucke eines liedes von Walther von der Vogelweide in den mund zu legen, statt sich an Heinrichs lieder zu halten? Die frage wurde am einfachsten zu beantworten sein durch die annahme, dass er das fragliche lied eben für Morungens eigentum gehalten' habe. Nun zeigt dasselbe wirklich gewisse Ubereinstimmungen mit Morungenschen liedern. Der schon von Grimm mit bertlcksichtigte vers der im Moringerlied aus Walther entlehnten strophe so wil (Var.: muoz) ich aber singen als $\hat{e}$ (Walther 72, 32 nu muoz ich singen aber als $\hat{e}$ ) stimmt zu Morungen 128, 14 ich wil singen aber als $\hat{e}$ noch besser als zu der von Grimm angefuhrten stelle. Die in eben jener strophe des Moringerliedes enthaltene und ebenfalls Walther entlelinte scherzhafte aufforderung des Moringers an den jungen, ihn an der alten braut zu răchen, ist bei Walther nach Wilmanns bemerkung (z. Walther 73,17 ) augenscheinlich parodie einer uberzarten strophe Heinrichs von Morungen, in welcher dieser die hoffnung ausspricht, sein sohn möge dereinst noch so schön werden, dass er die gegen den vater so bartherzige frouwe $z u$ herzbrechender liebessehnsucht entflammen und so ihn an der sprōden răchen werde. Die strophenform des Waltherschen gedichtes stimmt in der gestalt, welche ihr das Moringerlied gibt, genau uberein mit dem oben schon angezogenen tone Morungens MF 137, 10-16. Bei solchen beruhrungspunkten wäre es leicht erklärlich, wenn gerade dies lied Walthers dem Heinrich von Morungen zugeschrieben wăre, 
wie denn auch bei einem andern liede (MF 146, $11 \mathrm{ff}$.) die uberlieferung zwischen eben diesen beiden dichtern schwankt. Wir könnten schon aus allen den bisher erörterten umstănden schliessen, dass der verfasser des gedichtes vom Moringer jenes minnelied, welches er benutzte und teilweise dem Moringer in den mund legte, für das eigentun Heinrichs von Morungen gohalten habe.

Aber wir haben für diese annahme noch einen anderen, ganz schlagenden grund. Der dichter lernte das fragliche lied aus einer handschrift kennen, in welcher es unter Heinrichs von Morungen namen stand, oder, vorsichtiger ausgedruckt, in welcher es so aufgezeichnet war, dass man es fur Morungens eigentum halten konnte, ja halten musste, wenn man uberhaupt einen autornamen fur dasselbe haben wollte. Zum beweise dessen stelle ich zunăchst den beiden strophen des Moringerliedes die entsprechenden stlicke des Lachmannschen Walthertextes gegenuber.

\section{Moringerlied (Uhland):}

30 Ein langes schweigen hab ich gedacht, so wil ich aber singen als $\hat{e}$.

darzu hant mich die frawen bracht: die mugen mir wol gebieten mê. so bitt ich dich, da junger man, rich mich an der alten braut und schlach mit summerlatten an.

31 Was ich schaff so bin ich alt, davon so junget sie nit vil, dass mir mein bart ist graw gestalt, des sie ein jungen haben wil.

\section{W al ther (Lachmann):}

72, 31 Lange swigen des hat ich gedâht: nu muoz ich singen aber als 8 . darzuo hânt mich guote liute brâbt: die mugen mir wol gebieten mê.

73, 21 sô helfe iu got, hêr junger man, 80 rechet mich und gêt ir alten hât mit sumerlaten an.

Varianten des Moringerliedes: 30, 1 het ich mir. 2 muss. 3 die schœuen fr. 
73,17 Sol ich in ir dienste werden alt, die wile junget si niht vil. so ist mîn hâr vil lîhte alsô gestalt, dazs einen jungen danne wil.

Die verănderungen, welche Walthers lied erlitten bat, sind teilweise durch den neuen zusammenhang bedingt, in welchen dasselbe hier gebracht wurde. Aber nicht bei allen ist das der fall. Sie ruhren gar nicht durchweg vom dichter des Moringerliedes her, sondern finden sich zum guten teil schon in einer recension des Waltherschen liedes, welche von Lachmanns wesentlich auf die Heidelberger und Pariser handschriften gegrundetem texte erheblich abweicht. Diese recension liegt in der Weingartner handschrift vor. Man vergleiche nachstehende abweichungen dieser handschrift von Lachmanns text mit den entsprechenden stellen des Moringerliedes unter zuziehung der angegebenen varianten derselben. Walth. 12, 31 Langes swigen het ich mir gedaht - Mor. 30, 1 mit var. W. 72, 33 darzi hant mich schoene vrowen braht $=$ Mor. 30, 3 mit var. - W. 73, 22 das ir mich rechent an der alt en brut und slaht mit sumerlatten dran $=$ Mor. 30,6.7. W. 73, 17 bin ich in ir dienste worden alt $=$ Mor. 31, 1. W. 73, 18 da bi so $=$ Mor. 31, 2. - W. 73, 20 das si ainen jungen haben wil $=$ Mor. 31, 4. An allen diesen stellen stimmt das Moringerlied allein mit der Weingartner handschrift gegen alle ubrigen handschriften uberein; es ist also zweifellos, dass der verfasser desselben die Weingartner recension vor sich hatte. In der Weingartner handschr. aber ist das lied nicht unter Walthers namen uberliefert. Es bildet den schluss einer liedergruppe, welche ohne noue uberschrift auf die unter Heinrich von Morungen stebenden lieder folgt (Weingartner liederhandschrift herausg. von Pfeiffer s. 96 anm.). Ich denke, es bedarf keines weiteren beweises fur die tateache, dass der dichter des Moringerliedes das mehrfach von ibm benutzte und teilweise als des Moringers gesang widergegebene minnelied fur das werk Heinrichs von Morungen ansah.

Es bleibt somit nur noch ein punkt aufzuklăren: die ubertragung des namens der junge Herr von Neifen auf den nebenbuhler des Moringers. Als jungling musste dieser rival erschei- 
nen infolge der herbeiziehung der pseudomorungenschen strophen, welche den jungen mann dem alternden sănger und der alten braut gegenuberstellen. So lag es denn nahe, dass der dichter, wenn er sich nach einem namen fur diese rolle umsah, den eines jüneren kunstgenossen des Morungers wăhlte; und ein solcher war Gottfried von Neifen. Das durfte schon zur erklärung ausreichen. Eine beziehung zwischen Gottfrieds lied von einem falschen verkleideten pilger (von Walhen fuor ein bilgerîn) und der Moringersage, wie Scherer sie Lgesch. 8.742 vermutete, vermag ich nicht zu erkennen. Dort wird ein pilger, den man für einen heuchler hält, rom hauswirt abgewiesen, hier wird einer, dessen eigenschaft als pilger niemand anzweifelt, vom torwärter und der hausfrau sebr freundlich aufgenommen; der derzeitige hausherr, der junge von Neifen, tritt bei dieser scene gar nicht auf, und es ware doch ein leichtes gewesen ihn hier heranzuziehen, wenn dem dichter jenes Neifensche lied uberhaupt vorgeschwebt bätte. Er wurde es dann gewiss ăhnlich verwertet haben wie dasjenige, welches er dem Moringer zuschrieb; aber nirgend findet sich ein anklang. Eher kōnnte man vermuten, dass schon der dichter ebenso wie Konrad Grunenberg den Heinrich von Morungen fur einen Schwaben hielt, ihn deshalb Moringer nannte und ihm ebendeshalb gerade den schwäbischen minnesänger v. Neifen gegenuberstellte. Ja, es wäre dann die oben zugestandene möglichkeit nicht ausgeschlossen, dass er von jener durch Thomann bezeugten frau von Neufen, genannt Moringerin, gewusst und darin noch eine ganz besondere veranlassung gefunden hätte, den namen Neifen dem Moringer gegenubeizustellen und schliesslich beide sogar in einen verwantschaftschaftlichen zusammenhang zu bringen. Aber das bleibt eine unsichere combination. Vergessen darf auch nicht werden, dass noch bis ins 16. jahrhundert hinein neben jener tradition, welche den Heinrich von Morungen zu einem Schwaben machte, noch die richtigere uberlieferung fortbestand, nach welcher or zu Leipzig wohnte und starb. Der dichter brauchte vom herrn von Neifen nichts weiter zu wissen, als dass er ein minnesinger war und später als Heinrich von Morungen dichtete. Dadurch konnte er schon darauf verfallen, ihm die rolle des jungen nebenbuhlers zuzuweisen. 
Wir haben somit auf die oben aufgestellten fragen folgende antworten gefunden:

1. Der edele Moringer ist der minnesinger Heinrich von Morungen.

2. Der junge herr von Neifen ist der minnesinger Gottfried von Neifen.

3. Man thertrug auf Heinrich von Morungen die im eingang bestimmte sage, und zwar die sage in der besonderen form der legende vom frommen Thomasverehrer deshalb, weil Heinrich von Morungen sich den ruf eines eifrigen verehrers des heiligen Thomas verschafft hatte; vielleicht auch weil er überdies noch eine mit besonderen umständen verknupfte orientíahrt Dietrichs von Meissen mitmachte oder mitgemacht haben sollte.

4. Der spielmann, welcher das lied vom Moringer dichtete und bei seinem helden noch den minnesinger Heinrich von Morungen im sinne hatte, legte ihm ein lied Walthers von der Vogelweide in den mund, weil er dasselbe fur Morungens eigentum hielt, unter dessen namen er es nachweislich vorfand.

KIEL, d. 27 januar 1887.

F. VOGT. 